\title{
An Investigation to Compare the Numerical Model Between Shell and Solid for Honeycomb Light-weighted Mirror
}

\author{
Yu-Chuan Lin ${ }^{1,}$, Long-Jeng Lee ${ }^{1, b}$, Shenq-Tsong Chang ${ }^{1, c}$, \\ Yu-Cheng Cheng ${ }^{1, d}$ and Ting-Ming Huang ${ }^{1, e}$ \\ ${ }^{1}$ Instrument Technology Research Center, National Applied Research Laboratories, \\ 300 Hsinchu, Taiwan
}

ayclin@itrc.org.tw, ${ }^{b}$ ljlee@itrc.org.tw, ${ }^{c}$ stc@itrc.org.tw, ${ }^{d}$ yucheng@itrc.org.tw, ${ }^{e}$ tmw@itrc.org.tw

\begin{abstract}
Keywords: Numerical Modeling, Opto-mechanical Analysis, Light-weight Mirror
\end{abstract}
\begin{abstract}
This article reports an accurate analysis approach of finite element modeling and optical modeling. It has been used for design and analysis of many opto-mechanical systems such as large telescopes and laser systems. The approach is to represent mirror surface deformation derived from finite element analysis (FEA) by Zernike polynomials, such that the impact of deformation on optical system performance can be evaluated by optical design and analysis program. The methodology of shell-based and solid-based finite element modeling and the comparison of their results have been described in this paper. The result shows that the deformation of the light-weighted mirror designed by this approach fulfills the requirements of optical design. The simulation results of shell based FE model are in good agreement with those of solid based FE model.
\end{abstract}

\section{Introduction}

Large optical light-weighted mirrors have been increasingly applied in space-based telescope and high-energy laser applications. The Cassegrain telescope system with a primary mirror (M1), a secondary mirror (M2), and four-element correction lenses collects the images from ground target to the focal plane. The telescope configuration is shown in Fig.1. In the telescope, the light-weighted mirror plays a critical role both in quality of images and weight reduction of satellite payload due to launch vehicle constraint. For the high-energy laser systems, the weight of primary mirror will be even larger than the other components of full system. Therefore, because of the difficulty in supporting and handling, this large mirror must be light-weighted. The light-weighted mirror have been design and fabricated with various core-cells [1-9]. The popular designs were categorized as contoured back, open back pocket milled, sandwich, and actuated. Furthermore, the sectional forms of the light-weighted cell include triangle, rectangle, honeycomb, and even the combination of various shapes. The quilting deflection is an important issue for the selection of light-weighted cell that is related to the optimization design of a mirror. The quilting deflection due to polishing pressure is the minimum with honeycomb cell that compared to others light-weighted cell especially as the case of Zerodur mirror [3]. In this article, considering overall optical performance and manufacturing discussed in previous study $[1,8,9]$, the mirror with honeycomb cell and open back structure were selected.

Light-weight design and analysis of mirrors are very important issues for weight reduction and better performance of system. The FEA is a useful technique for obtaining approximate numerical solutions to predict the response of engineering system subjected to external influences. Usually, the FEA model type, such as beam, plane, shell and solid, needs to be changed according to the analysis requirements. In order to reduce the computational time and cost, the shell-based FE model (reduced model) has been developed and discussed in this paper, which considers the natural frequency and mirror surface deformation induced by gravity load. In general, the optical performance of mirror can 
be illustrated by the terms of Zernike polynomials which provide the parameters of optical aberrations such as piston, tilt, defocus, coma etc. [1-4]. The finite element analysis combined with Zernike polynomials fitting were successfully used for optimum design of optical mirror in the previous studies [8,9]. In order to compare the results between solid and shell-base model and make sure which model can provide the more confident result, this article also process this kind of integrated opto-mechanical analysis applied to both models. The results exhibit that these models give close results of mechanical properties and their optical performances from Zernike polynomials fitting meet the tolerance of optical requirement.

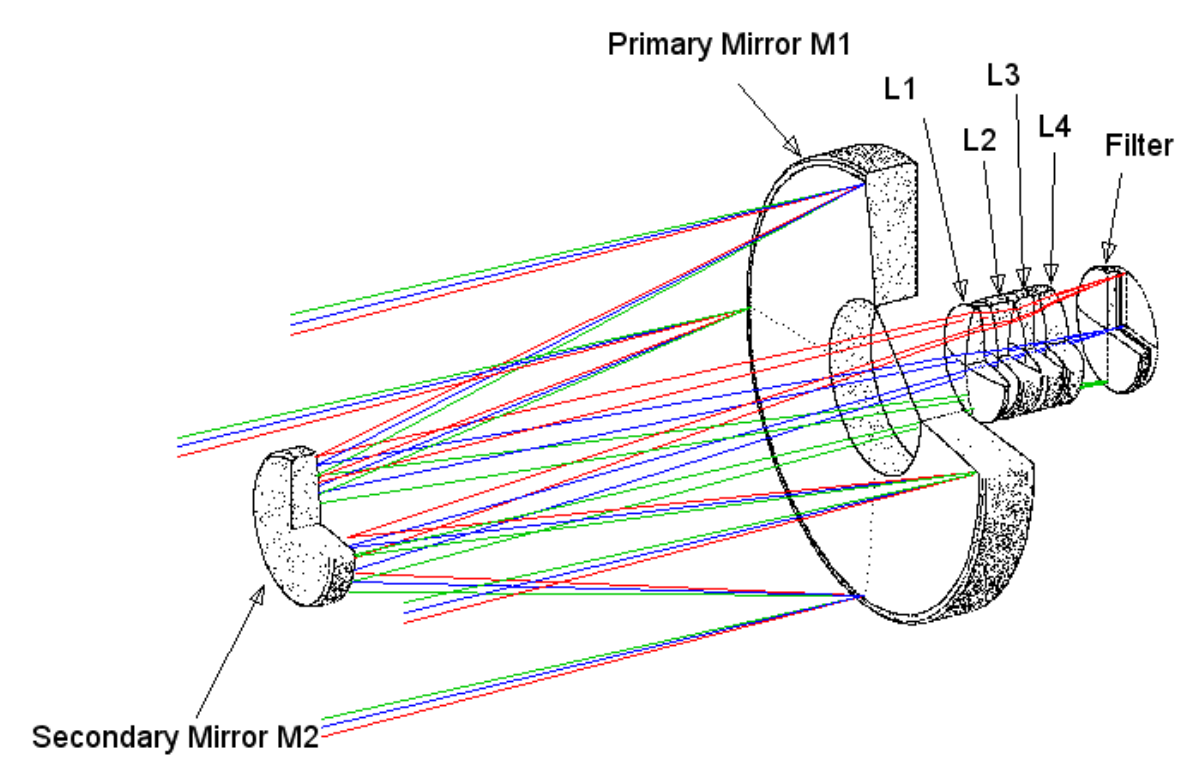

Fig. 1 Cassegrain system configuration

\section{Design and Analysis Process}

The Cassegrain telescope system has an aspheric primary mirror, an aspheric secondary mirror and a four-element corrector lens. The telescope images a portion of ground target on the focal plane. The primary mirror has been designed as shown in the Fig.2. The light-weighted primary mirror has cell with the rib thickness of $5 \mathrm{~mm}$ and the inscribed circle of $45 \mathrm{~mm}$. The light-weight process removes $52 \%$ weight of the original solid mirror in same dimension. The light-weighted mirror requires integrated opto-mechanical analysis to predict the optical performance. The analysis approach allows FEA results being accurately transferred to optical analysis software for the further analysis of optical performance. In general, the format of FEA data is not acceptable for optical analysis software, and the displacements and stresses at arbitrary located finite element nodes can not be input directly into optical software. Therefore, the computed displacement data provided by FEA codes must be translated into forms acceptable by optical software [1]. In this article, interface codes had been developed, which converts FEA of the mechanical results from ANSYS to optical analysis software CODEV. The code to transfer finite element node displacement to sag-displacement, or normaldisplacement for the mirror surface was written based on EXCEL, and the program to fit the deformed mirror surface to Zernike polynomial coefficients was written based on MatLab. 


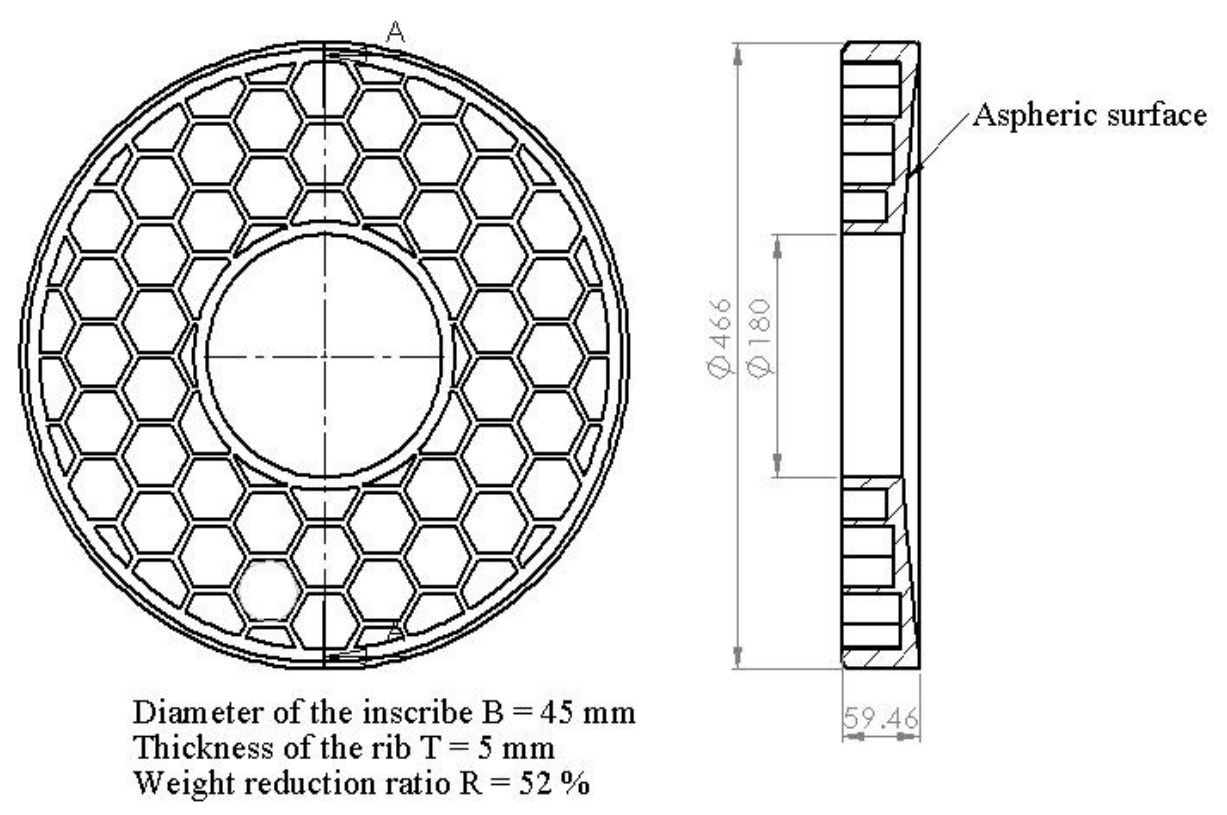

Fig.2 Light-weighted of the primary mirror

\section{Results and Discussion}

The selection of numerical model in FEA is depended on the engineering problem as the change of spatial dimension. The range of spatial dimension in FEA including four types such as one-dimension, two-dimension, three-dimension and Axial-symmetry. In the rule of thumb, FEA use as simple model as possible to represent engineering problem without losing accuracy of results. Therefore, the shell-based and solid-based FE models of the primary mirror have been established for comparison. The corresponding finite element models of both types are shown in Fig. 3 and Fig. 4. For the convergence of the solid model, the FEA model needs 113,382 nodes and 61,711 elements included in this analysis, and the elements have mid-side nodes as 20 -noded solid. The shell model contains 4,235 nodes and 4,408 elements, and the elements have only corner nodes as 4-noded shell. The computational time of shell model is found to be reduced about $98.30 \%$ and its storage space in the computer had benefits of size reduction about $93.64 \%$ in the same condition. However, a critical limitation of shell model could not predict the more accuracy of lens deformation in the direction of optical axis, especially when the lens is thick. In the case of thin lenses or light-weighted mirrors, its shell model could exhibit the similar results as in solid model but with less computation time and storage space.

Fig. 5 shows the result of first order natural frequency and modal shape of the shell and solid model. The values of natural frequency are calculated at $677.36 \mathrm{~Hz}$ and $730.90 \mathrm{~Hz}$ respectively, and the first order modal shape almost agrees with each other. The result reveals solution of eigenvalue and eigenvector problems by using 4-noded shell and 20-noded solid FEA model are feasible approach. The two analysis cases of the mirror subject to $1 \mathrm{~g}$ load acting perpendicular to and along the optical axis are calculated to predict the maximum deformation of the primary mirror. Three joints are mounted on the outer edge of the primary mirror for both cases. Fig. 6 shows that the primary mirror predicted maximum deformation of the shell model under $1 \mathrm{~g}$ load perpendicular to the optical axial is $59 \mathrm{~nm}$ and under the same load condition the predicted maximum deformation of solid model is 48 $\mathrm{nm}$. The results agree to each other very well. Fig. 7 shows that the primary mirror predicted maximum deformation of the shell model under $1 \mathrm{~g}$ load along the optical axial is $218 \mathrm{~nm}$ and under the same load condition the solid model predicted maximum deformation is $211 \mathrm{~nm}$, and both also agree to each other very well. 


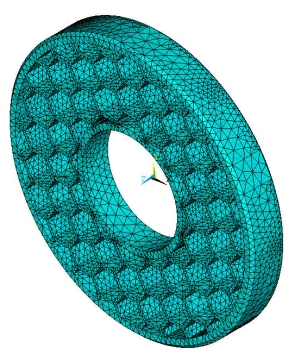

(a) Light-weighted surface

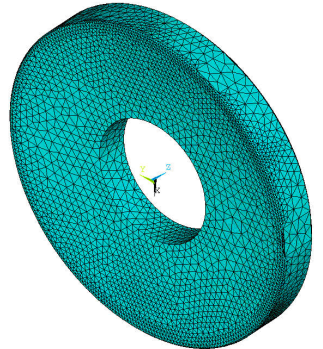

(b) Optical surface

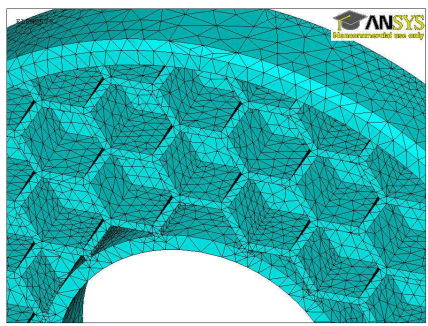

(c) Local mesh

Fig.3 Three-dimensional solid model of the light-weighted mirror

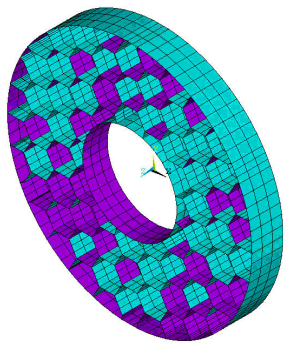

(a) Light-weighted surface

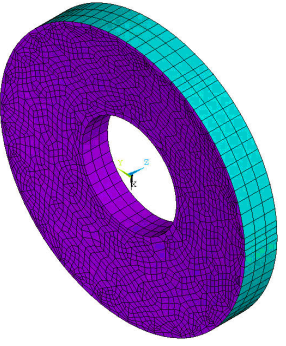

(b) Optical surface

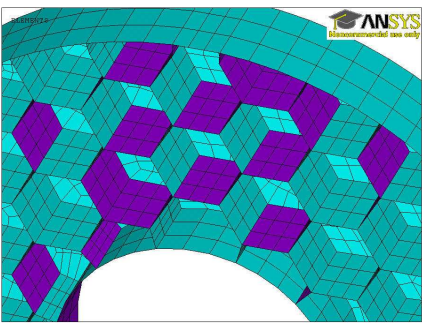

(c) Local mesh

Fig. 4 Three-dimensional shell model of the light-weighted mirror

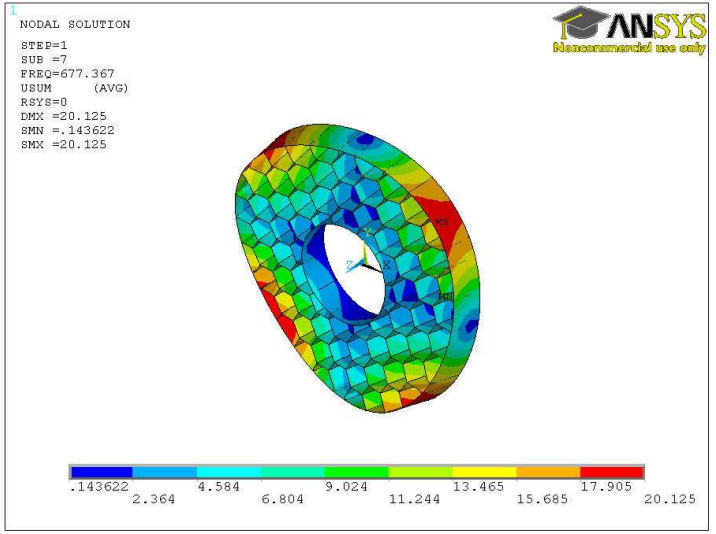

(a) Shell model

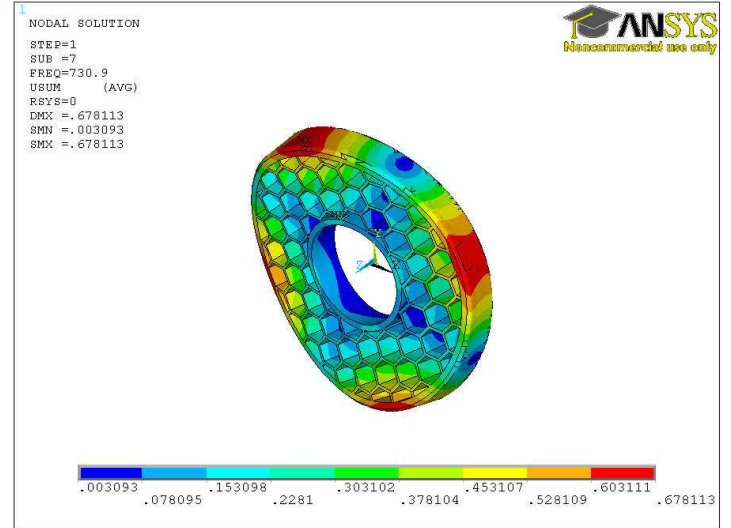

(b) Solid model

Fig. 5 A comparison of the results from shell and solid models for modal analysis

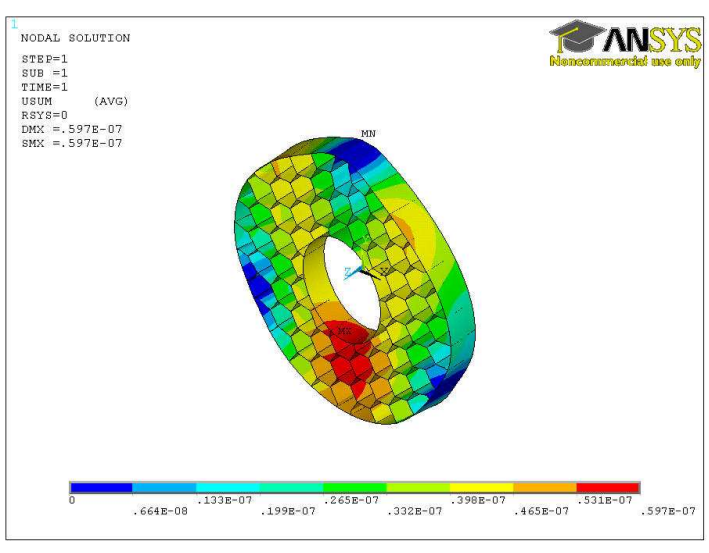

(a) Shell model

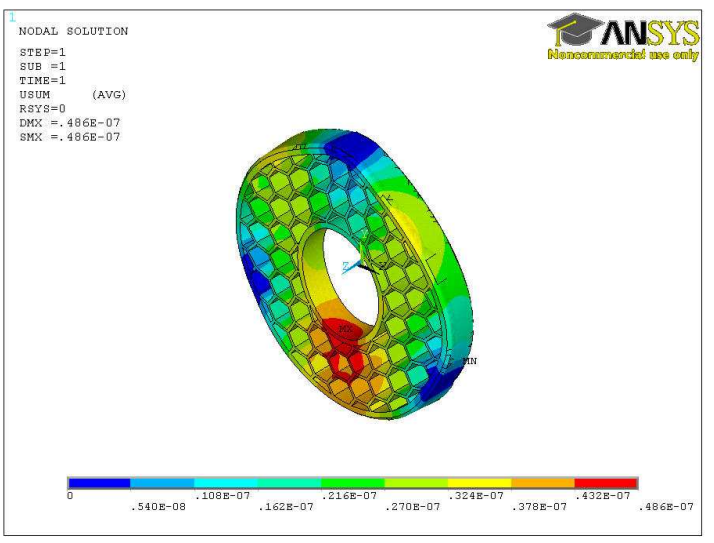

(b) Solid model

Fig. 6 A comparison of the results from shell and solid models subject to gravity acting perpendicular to the optical axis 


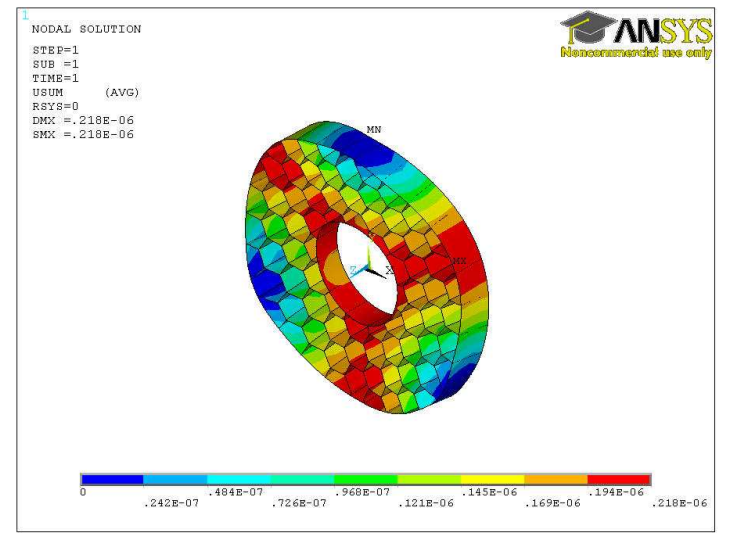

(a) Shell model

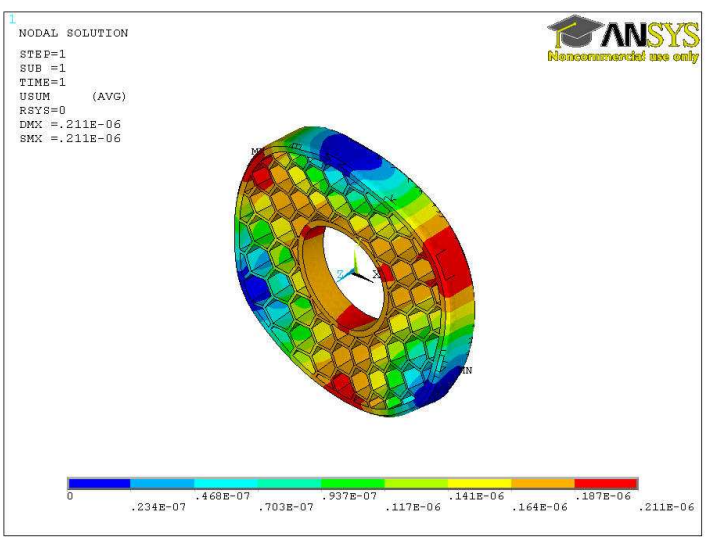

(b) Solid model

Fig. 7 A comparison of the results from shell and solid models subject to gravity acting along the optical axis

In general, the shape of an optical surface is typically described by the sag of the surface as the function of radial height. However, the sag deformation is not equal to the finite element approached Z-displacement due to the FEA node position may also has lateral displacement. Usually, the structural displacement data must take coordinate transformation to the optical coordinate system based upon sag expression. As in the optical performance of mirror, the terms of Zernike polynomial coefficients are generally useful in the interpretation of many types of optical operations such as defocus, coma and spherical aberration etc. Zernike polynomial fitting has been performed from the surface deformation of the FEA results as shown in Fig. 6 and 7. The fitting gives the terms of coefficients, peak to valley (P-V) values and root mean square (R.M.S) of its deformation of optical surface. The FEA deformation results of shell and solid model have been fitted and plotted by Zernike polynomials as shown in Fig. 8. Again, the Zernike polynomial fitting of the surface deformation results of shell model agrees with those of the solid model. As a summary, a comparison of the weight, center of mass and deformation results of shell and solid models due to gravity are shown in table1.

Shell
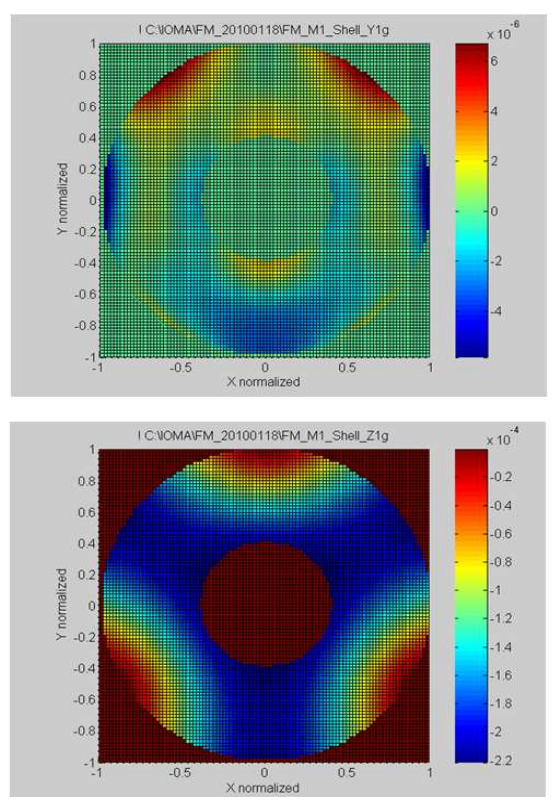

Solid
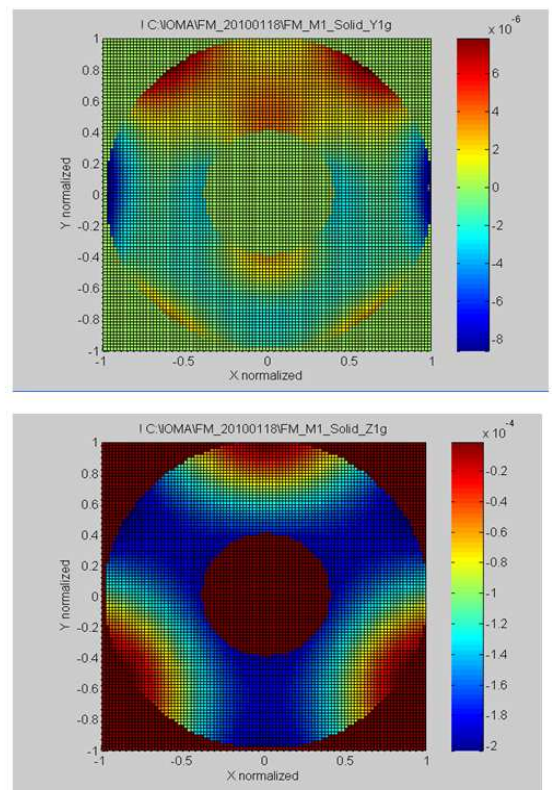

Diff
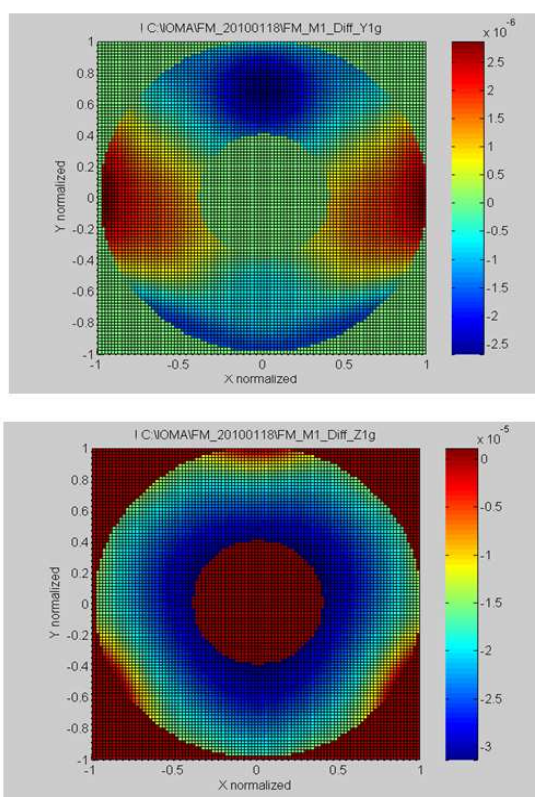

Fig. 8 A comparison of the results from shell and solid models for Zernike polynomials fitting 
Table.1 A comparison of the results between solid and shell models

\begin{tabular}{|c|c|c|}
\hline m1 model & Shell model & Solid model \\
\hline Mass $(\mathrm{Kg})$ & 9.48 & 9.42 \\
\hline Center of mass $(\mathrm{mm})$ & $0,0,12.16$ & $0,0,11.84$ \\
\hline $\begin{array}{c}\text { Free-Free mode } \\
\text { Eigenvalue }(\mathrm{Hz})\end{array}$ & 677.36 & 730.9 \\
\hline $\begin{array}{c}\text { Fixed-B.C. mode } \\
\text { Eigenvalue }(\mathrm{Hz})\end{array}$ & 1208 & 0.048 \\
\hline $\begin{array}{c}\text { Y-1gravity }(\mu \mathrm{m}) \\
\text { Max. Displacement }\end{array}$ & 0.059 & 0.211 \\
\hline $\begin{array}{c}\text { Z-1 gravity }(\mu \mathrm{m}) \\
\text { Max. Displacement }\end{array}$ & 0.129 & 0.177 \\
\hline $\begin{array}{c}\text { Y-1 gravity }(\mu \mathrm{m}) \\
\text { Peek to valley WFE }\end{array}$ & 0.022 & 0.020 \\
\hline $\begin{array}{c}\text { Z-1 gravity }(\mu \mathrm{m}) \\
\text { Peek to valley WFE }\end{array}$ & 0.218 & 1234 \\
\hline
\end{tabular}

\section{Conclusion}

The methodology of modeling the shell-based and its comparison to solid-based finite element model have been described in this paper. The simulation result of shell based FE model are in good agreement with solid based FE model, and the shell based model requires less memory and computational time. In addition, the finite element analysis combined with Zernike polynomials fitting had been successfully applied to the design of optical mirror. The efficiencies with little time consuming of calculation and lower processing memory, the shell based FE model give the similar result as in the case of solid based FE model and its optical analysis from Zernike polynomials fitting show the performance agreement to the optical requirement.

\section{References}

[1] D. Vukobratovich, In: Qptomechanical Design, edited by P. R. Yoder, SPIE Press, SD (1992), in press.

[2] P. R. Yoder, In: Qpto-Mechanical System Design, edited by B. J. Thompson, Marcel Dekker, Inc., NY (1993), in press.

[3] D. Vukobratovich, In: Handbook of Optomechanical Engineering, edited by A. Ahmad, CRC Press NY (1997), in press.

[4] K. B. Doyle, V. L. Genberg, and G. J. Michels, Integrated Optomechanical Analysis, edited by A. R. Weeks, SPIE Press, WA (2002), in press.

[5] Y. Y. Han, Y. M. Zhang, J. C. Han, J. H. Zhang, W. Yao and Y. F Zhou: Trans. Nonferrous Met. Soc. China, Vol.16 (2006), p.696.

[6] J. C. Han, Y. Y. Han, Y. M. Zhang, J. H. Zhang, W. Yao and Y. F Zhou: Trans. Nonferrous Met. Soc. China, Vol.17 (2007), p.1160.

[7] Y. Y. Han, Y. M. Zhang, J. C. Han, J. H. Zhang, W. Yao and Y. F Zhou: Journal Wuhan University of Technology--Mater. Sci. Ed., (2008), p.259.

[8] Y. C. Lin, L. J. Lee, S.T. Chang, T. M. Huang and C. H. Hwang: Design and Simulation of Lightweight Mirror for Space Application, Design, Test, Integration and Packaging of MEMS/MOEMS, (2009), p.334.

[9] Y. C. Lin, L. J. Lee, S.T. Chang, T. M. Huang and C. H. Hwang: Integrated Optomechanical Analysis of Large Optical Mirror for Space-based Telescope System, International Conference on Manufacturing and Engineering System, (2009), p.624. 